\title{
Coupled Sound and Heat Flow and the Method of Least Squares
}

\author{
By Alfred Carasso
}

\begin{abstract}
We construct and analyze a least-squares procedure for approximately solving the initial-value problem for the linearized equations of coupled sound and heat flow, in a bounded domain $\Omega$ in $R^{N}$, with homogeneous Dirichlet boundary conditions. The method is based on Crank-Nicolson time differencing. To approximately solve the resulting system of boundary value problems at each time step, a least-squares method is devised, using trial functions which need not satisfy the homogeneous boundary conditions. Certain unknown normal derivatives of the solution enter the boundary integrals. By using suitable weights, these unknown derivatives can be set equal to zero without impairing the $O\left(k^{2}\right)$ accuracy of the Crank-Nicolson scheme. However, one must use smoother trial functions to obtain this accuracy.
\end{abstract}

1. Introduction. In a recent paper, [4], Bramble and Thomée discuss least-squares methods in the numerical computation of the homogeneous Dirichlet problem for the heat equation in a bounded domain $\Omega$ in $R^{N}$. The technique is to first discretize the time variable, using an implicit scheme, to obtain a sequence of elliptic boundary value problems at each time step. This latter problem is then approximately solved by "least squares". The importance of this work, in the context of the approximation of timedependent problems by variational methods, rests in the fact that the trial functions need not satisfy the boundary conditions, so that one can treat problems in general domains.

While only the heat equation is discussed in [4], the ideas would seem to have application to other evolution equations. In the present paper, we consider a mixed initial-boundary value problem for a coupled system of two evolution equations, the linearized equations of coupled sound and heat flow, in a general domain $\Omega$ in $R^{N}$, with a smooth boundary $\partial \Omega$. This problem is the best known example of a class of problems important in the applications. Other concrete instances of coupled hyperbolicparabolic equations exist and are mentioned in [1], [6], [13], and [14]. A class of coupled equations is investigated in [7]. Mathematically, the problem involves consideration of two or more unbounded operators of different strength. Consequently, the study of the stability properties of explicit schemes (in one space dimension) has interested several authors. See [7], [8], [11] and [12]. In [17], a Crank-Nicolson Galerkin method is proposed for the one-dimensional problem, using trial functions satisfying

Received September 7, 1973.

AMS (MOS) subject classifications (1970). Primary 65M15. 
the boundary conditions. Since the semiboundedness of the spatial operator is then automatically preserved, there are no stability difficulties with this procedure.

Very little seems to have been published in connection with the above problem in more than one space dimension. In this paper, we analyze a least-squares procedure based on the Crank-Nicolson time discretization. Since the trial functions do not satisfy the boundary conditions, the problem of formulating the fully-discrete scheme so as to obtain stability, turns out to be rather interesting. While we follow closely the organization of [4], there are major differences both in the formulation of the fully discrete scheme and in the type of results we obtain. In the problem treated in [4], the analytic solution operator, $e^{t A}$, is a holomorphic semigroup satisfying the characteristic estimate

$$
\left\|A^{m} e^{t A}\right\|_{L^{2}} \leqslant C_{m} t^{-m}, \quad t>0, m=1,2, \cdots .
$$

This smoothing property of $e^{t A}$ plays an important role in several places in [4]. In particular, in the convergence theorems, [4, Theorems 5.1, 5.2], it is used explicitly to estimate the $L^{2}$ norm of the error at time $t$ with the order of the best approximation to the solution by functions in the approximating subspace. In the problem treated here, the solution operator is a $C_{0}$ semigroup, but not a holomorphic one, and we are not able to obtain the order of the best approximation for the $L^{2}$ norm of the error at time $t$. Beyond that, other difficulties arise inevitably, due to the fact that one has a coupled system of boundary value problems at each time step rather than a single elliptic problem. In constructing the norm in which to approximate the solution at each time step, we find that we must include more boundary data than are actually supplied in the analytic problem. For example, although only the value of the temperaturezero-is prescribed on $\partial \Omega$, one needs both the temperature and its Laplacian in the boundary integral, due to the fact that operators of different strength are involved. We show that if the initial data are sufficiently smooth, the Laplacian of the temperature is also zero on $\partial \Omega$, so that these extra data are actually known. More serious is another coupling effect, originating in the hyperbolic problem, which forces us to include the normal derivatives of certain components of the solution in the boundary integral.

These extra data are unknown. We show that by using suitable weights, these derivatives can be taken to be zero without impairing the $O\left(k^{2}\right)$ accuracy of the Crank-Nicolson scheme. A penalty must be paid for being able to use such wrong values. It turns out that one must employ smoother trial functions than in [4], in order to obtain the same accuracy. See the remarks after Theorem 5.1.

We use the letter $C$ to denote a generic constant.

2. The Analytic Problem. We consider the infinitesimal motions of a compressible fluid in which the transfer of energy by thermal conduction is a significant aspect of the flow. Let $p_{0}, E_{0}, V_{0}$ and $\mathbf{u}_{0}$, respectively, denote ambient values of the pressure, specific internal energy, specific volume and material velocity, and let $p, E, V$ and $\mathbf{u}$ denote small deviations from these quantities. Let $c=\sqrt{p_{0} V_{0}}$ be the isothermal sound 
speed, $\gamma>1$ be the ratio of specific heats, and $\sigma>0$ the thermal conductivity. In terms of the auxiliary variables $w=c V / V_{0}, e=(\gamma-1) E / c$, the linearized equations expressing conservation of mass, momentum, and energy, are, cf. [6] and [13],

$$
\begin{gathered}
\partial w / \partial t=c \nabla \cdot \mathbf{u}, \\
\partial \mathbf{u} / \partial t=c \nabla w-c \nabla e, \\
\partial e / \partial t=\sigma \Delta e-(\gamma-1) c \nabla \cdot \mathbf{u} .
\end{gathered}
$$

We assume the disturbance confined to a fixed spatial domain $\Omega$, in $R^{N}$, and that, for simplicity, the ambient conditions prevail on the smooth boundary $\partial \Omega$ of $\Omega$, so that $e=w=0$ on $\partial \Omega$, for all $t \geqslant 0$. Taking the divergence of both sides in (2.2) and eliminating $\nabla \cdot \mathbf{u}$ from the resulting system, we obtain two equations for the unknown scalar fields $w(x, t)$ and $e(x, t)$, namely,

$$
\begin{aligned}
w_{t t} & =c^{2} \Delta w-c^{2} \Delta e, \quad x \in \Omega, t>0, \\
e_{t} & =\sigma \Delta e-(\gamma-1) w_{t}, \quad x \in \Omega, t>0,
\end{aligned}
$$

together with,

$$
e=w=0, \quad x \in \partial \Omega, t \geqslant 0 .
$$

We shall show that, given initial values of $e, w$ and $w_{t}$, the resulting initial-boundary value problem is well posed in an appropriate function space. It is convenient to make use of the spaces $\dot{H}^{s}, s \geqslant 0$, introduced in [4]. Let $\left\{\lambda_{m}\right\}_{m=1}^{\infty}$ be the eigenvalues of the negative Laplacian in $\Omega$, with zero Dirichlet data on $\partial \Omega$, and let $\left\{\phi_{m}\right\}_{m=1}^{\infty}$ be the corresponding orthonormal sequence of eigenfunctions. For a given $v \in L^{2}(\Omega)$, let $\left\{\beta_{m}\right\}_{m=1}^{\infty}$ be the sequence of Fourier coefficients of $v$ relative to the $\left\{\phi_{m}\right\}$. For each $s \geqslant 0$, the Hilbert space $\dot{H}^{s}$ is defined to be the subspace of $L^{2}(\Omega)$ consisting of all $v$ 's for which the norm

$$
\|v\|_{s}=\left(\sum_{m=1}^{\infty} \lambda_{m}^{s}\left|\beta_{m}\right|^{2}\right)^{1 / 2}
$$

is finite. $\dot{H}^{\infty}=\bigcap_{s>0} \dot{H}^{s}$ is dense in every $\dot{H}^{s}$ and consists of all $C^{\infty}(\bar{\Omega})$ functions which vanish on $\partial \Omega$ together with all powers of their Laplacian. If $s$ is a nonnegative integer, and $v \in \dot{H}^{\infty}$, the $s$-norm is equivalent to the usual Sobolev norm, $\|v\|_{H^{s}}$. It follows from the trace theorems, see, e.g., [10], that if $s \geqslant 1$ and $v \in \dot{H}^{s}$, then $v=0$ on $\partial \Omega$. If $v \in \dot{H}^{s}, s \geqslant 3$, then both $v$ and $\Delta v$ vanish on $\partial \Omega$.

Returning to (2.4), (2.5), put $w_{t}=v$ in (2.4), (2.5), and let $G$ be the $3 \times 3$ matrix

$$
G=\left[\begin{array}{ccc}
0 & I & 0 \\
c^{2} \Delta & 0 & -c^{2} \Delta \\
0 & -(\gamma-1) I & \sigma \Delta
\end{array}\right]
$$

Let $\hat{G}=\hat{G}\left(\lambda_{m}\right)$ be the $3 \times 3$ matrix 


$$
\hat{G}=\left[\begin{array}{ccc}
0 & 1 & 0 \\
-c^{2} \lambda_{m} & 0 & c^{2} \lambda_{m} \\
0 & (1-\gamma) & -\sigma \lambda_{m}
\end{array}\right] .
$$

Let $U$ be the three component vector $U=[w, v, e]^{T}$. The initial-boundary value problem for (2.4), (2.5) may be equivalently written as

$$
\begin{aligned}
U_{t} & =G U, \quad x \in \Omega, t>0, \\
U(x, 0) & =[f(x), g(x), h(x)]^{T}, \quad x \in \Omega, \\
e & =w=0, \quad x \in \partial \Omega, t \geqslant 0 .
\end{aligned}
$$

We now introduce the Hilbert space $\dot{H}^{s}=\dot{H}^{s+1} \times \dot{H}^{s} \times \dot{H}^{s+1}, s \geqslant 0$, consisting of all three component vectors $U(x)$, for which the norm

$$
\|U\|_{s}=\left\{c^{2}\|w\|_{s+1}^{2}+\|v\|_{s}^{2}+\frac{c^{2}}{\gamma-1}\|e\|_{s+1}^{2}\right\}^{1 / 2}
$$

is finite. In general, the components of $U$ will depend on both $x$ and $t$. We set

$$
\|U(t)\|_{s}^{2}=c^{2}\|w(\cdot, t)\|_{s+1}^{2}+\|v(\cdot, t)\|_{s}^{2}+\frac{c^{2}}{\gamma-1}\|e(\cdot, t)\|_{s+1}^{2} .
$$

We have the following

THEOREM 2.1. Let $l \geqslant 0$. For each initial value $U(x, 0) \in \dot{H}^{l}$, problem $(2.10)-$ (2.12) has a unique solution and the following estimate holds:

$$
\|U(t)\|_{l} \leqslant\|U(0)\|_{l} .
$$

Proof. We construct the solution by expanding in the eigenfunctions of $-\Delta$. Let

$$
U(x, 0)=\left[\sum_{m=1}^{\infty} a_{m} \phi_{m}, \sum_{m=1}^{\infty} b_{m} \phi_{m}, \sum_{m=1}^{\infty} d_{m} \phi_{m}\right]^{T},
$$

and put

$$
U(x, t)=\left[\sum \alpha_{m}(t) \phi_{m}, \sum \beta_{m}(t) \phi_{m}, \sum \delta_{m}(t) \phi_{m}\right]^{T} .
$$

Substitution of (2.17) in (2.10) leads to an initial-value problem for a linear system of ordinary differential equations, for each fixed $m$, namely

$$
\left[\dot{\alpha}_{m}(t), \dot{\beta}_{m}(t), \dot{\delta}_{m}(t)\right]^{T}=\hat{G}\left(\lambda_{m}\right)\left[\alpha_{m}(t), \beta_{m}(t), \delta_{m}(t)\right]^{T},
$$

with

$$
\left[\alpha_{m}(0), \beta_{m}(0), \delta_{m}(0)\right]^{T}=\left[a_{m}, b_{m}, d_{m}\right]^{T} .
$$

For each fixed $m$, equip three-dimensional Euclidean space with the inner product

$$
(x, y)=c^{2} \lambda_{m} x_{1} \bar{y}_{1}+x_{2} \bar{y}_{2}+\frac{c^{2} \lambda_{m}}{\gamma-1} x_{3} \bar{y}_{3} .
$$

Then, for each $m$, the matrix $\hat{G}\left(\lambda_{m}\right)$ is dissipative, i.e.,

$$
\operatorname{Re}(\hat{G} x, x) \leqslant 0 .
$$


It follows immediately from (2.21), that (2.18), (2.19) has a unique solution, and

$$
\begin{aligned}
c^{2} \lambda_{m}\left|\alpha_{m}(t)\right|^{2}+ & \left|\beta_{m}(t)\right|^{2}+\frac{c^{2} \lambda_{m}}{\gamma-1}\left|\delta_{m}(t)\right|^{2} \\
& \leqslant c^{2} \lambda_{m}\left|a_{m}\right|^{2}+\left|b_{m}\right|^{2}+\frac{c^{2} \lambda_{m}}{\gamma-1}\left|d_{m}\right|^{2},
\end{aligned}
$$

for each $m$. The estimate (2.15) now follows from (2.22) and (2.14). This proves the theorem.

3. The Semidiscrete Problem. Let $k>0$ be a small increment in the time variable. Leaving the space variables continuous, we discretize the time in (2.10), using the Crank-Nicolson scheme, to obtain a sequence of boundary value problems at each time step $n k$, namely,

$$
\begin{aligned}
(I-k G / 2) W^{n+1} & =(I+k G / 2) W^{n}, \quad x \in \Omega, n=0,1,2, \cdots, \\
w_{1}^{n+1} & =w_{3}^{n+1}=0, \quad x \in \partial \Omega, \\
W^{0} & =U(x, 0), \quad x \in \Omega .
\end{aligned}
$$

Here $W^{n}(x)=\left[w_{1}^{n}(x), w_{2}^{n}(x), w_{3}^{n}(x)\right]^{T}$ is a three-component vector which presumably will approximate $U(x, n k)$, where $U(x, t)$ is the solution to (2.10)-(2.12). This discrete initial-value problem is also well posed.

THEOREM 3.1. Fix $s \geqslant 0$ and let $W^{0} \in \dot{H}^{s}$. Then, there exists a unique solution, $\left\{W^{n}\right\}$, of (3.1)-(3.3) and

$$
\left\|W^{n}\right\|_{s} \leqslant\left\|W^{0}\right\|_{s}, \quad n=0,1,2, \cdots .
$$

Proof. We may again construct $\left\{W^{n}\right\}$ by expanding in the eigenfunctions of $-\Delta$. Put and let

$$
W^{n}=\left[\sum_{m} \alpha_{m}^{n} \phi_{m}, \sum \beta_{m}^{n} \phi_{m}, \sum \delta_{m}^{n} \phi_{m}\right]^{T}
$$

$$
y^{n}=\left[\alpha_{m}^{n}, \beta_{m}^{n}, \delta_{m}^{n}\right]^{T}
$$

Then, for each fixed $m$, the coefficients $y^{n}$ satisfy

$$
[I-k \hat{G} / 2] y^{n+1}=[I+k \hat{G} / 2] y^{n}, \quad n=0,1,2, \cdots .
$$

We again use the scalar product (2.20) for the space of vectors $y^{n}$. Forming the scalar product on both sides of (3.7) with the vector $y^{n+1}+y^{n}$, we get

$$
\begin{aligned}
\left\|y^{n+1}\right\|^{2}-\left\|y^{n}\right\|^{2}-\left(y^{n}, y^{n+1}\right)+\left(y^{n+1}, y^{n}\right) \\
=(k / 2)\left(\hat{G}\left(y^{n+1}+y^{n}\right),\left(y^{n+1}+y^{n}\right)\right) .
\end{aligned}
$$

Using (2.21) in (3.8), we have

$$
\left\|y^{n+1}\right\| \leqslant\left\|y^{n}\right\| \text {. }
$$

This proves that (3.1)-(3.3) has a unique solution and establishes the estimate (3.4). 
Lemma 3.1. Let $y=\left[y_{1}, y_{2}, y_{3}\right]^{T}$ be a vector in three-dimensional space. Then, there is a constant $C$, independent of $y$ and $m$, such that, in the norm induced by (2.20),

$$
\begin{aligned}
\| e^{k \hat{G}} y-(I-k \hat{G} / 2)^{-1}(I & +k \hat{G} / 2) y \| \\
& \leqslant\left\|(I-k \hat{G} / 2) e^{k \hat{G}} y-(I+k \hat{G} / 2) y\right\| \\
& \leqslant C k^{3}\left\{\lambda_{m}^{5}\left|y_{1}\right|^{2}+\lambda_{m}^{5}\left|y_{2}\right|^{2}+\lambda_{m}^{7}\left|y_{3}\right|^{2}\right\}^{1 / 2} .
\end{aligned}
$$

Proof. To begin with, since $\operatorname{Re}(\hat{G} y, y) \leqslant 0$, we have $\left\|(I-k \hat{G} / 2)^{-1}\right\| \leqslant 1$, so that

$$
\left\|(I-k \hat{G} / 2)^{-1} z\right\| \leqslant\|z\| .
$$

If we now apply (3.11) to the vector

$$
z=(I-k \hat{G} / 2) e^{k \hat{G}} y-(I+k \hat{G} / 2) y,
$$

we obtain the first inequality in (3.10).

Next, by Taylor's theorem, for some $\tau$ with $0<\tau<k$,

$$
(I-k \hat{G} / 2) e^{k \hat{G}}=I+k \hat{G} / 2-\left(\tau k^{2} / 4\right) \hat{G}^{3} e^{\tau \hat{G}} .
$$

Hence, for any vector $y$,

$$
\left\|(I-k \hat{G} / 2) e^{k \hat{G}} y-(I+k \hat{G} / 2) y\right\| \leqslant\left(k^{3} / 4\right)\left\|e^{\tau \hat{G}} \hat{G}^{3} y\right\| \leqslant\left(k^{3} / 4\right)\left\|\hat{G}^{3} y\right\|,
$$

since $\left\|e^{\tau \hat{G}}\right\| \leqslant 1$, independently of $m$, due to the dissipativity of $\hat{G}\left(\lambda_{m}\right)$.

It remains to calculate $\left\|\hat{G}^{3} y\right\|$. We have

$$
\hat{G}^{3}=\left[\begin{array}{ccc}
0, & -\gamma c^{2} \lambda_{m}, & -\sigma c^{2} \lambda_{m}^{2} \\
\gamma c^{4} \lambda_{m}^{2}, & (\gamma-1) \sigma c^{2} \lambda_{m}^{2}, & \sigma^{2} c^{2} \lambda_{m}^{3}-\gamma c^{4} \lambda_{m}^{2} \\
(1-\gamma) c^{2} \sigma \lambda_{m}^{2}, & \gamma(\gamma-1) c^{2} \lambda_{m}-\sigma^{2}(\gamma-1) \lambda_{m}^{2}, & 2 c^{2} \sigma(\gamma-1) \lambda_{m}^{2}-\sigma^{3} \lambda_{m}^{3}
\end{array}\right] .
$$

Hence,

$$
\begin{aligned}
\left\|\hat{G}^{3} y\right\|^{2}= & c^{2} \lambda_{m}\left|c^{2} \gamma \lambda_{m} y_{2}+c^{2} \sigma \lambda_{m}^{2} y_{3}\right|^{2} \\
& +\mid \gamma c^{4} \lambda_{m}^{2} y_{1}+(\gamma-1) \sigma c^{2} \lambda_{m}^{2} y_{2} \\
& +\sigma^{2} c^{2} \lambda_{m}^{3} y_{3}-\left.\gamma c^{4} \lambda_{m}^{2} y_{3}\right|^{2} \\
& +\frac{c^{2} \lambda_{m}}{\gamma-1} \mid(1-\gamma) c^{2} \sigma \lambda_{m}^{2} y_{1}+\gamma(\gamma-1) c^{2} \lambda_{m} y_{2}-\sigma^{2}(\gamma-1) \lambda_{m}^{2} y_{2} \\
& +2 c^{2} \sigma(\gamma-1) \lambda_{m}^{2} y_{3}-\left.\sigma^{3} \lambda_{m}^{3} y_{3}\right|^{2}
\end{aligned}
$$

Since $\lambda_{m} \uparrow \infty$, we have from (3.16), with a constant $C$ independent of $m$,

$$
\left\|\hat{G}^{3} y\right\|^{2} \leqslant C\left\{\lambda_{m}^{5}\left|y_{1}\right|^{2}+\lambda_{m}^{5}\left|y_{2}\right|^{2}+\lambda_{m}^{7}\left|y_{3}\right|^{2}\right\} .
$$

The lemma follows from (3.14) and (3.17).

We denote by $E(t)$ the solution operator at time $t$ associated with the analytic initial-boundary value problem (2.10)-(2.12), and by $E_{k}(t)$ the solution operator associated with the semidiscrete problem (3.1)-(3.3). Both of these operators are bounded from $H^{s}$ into itself, $s \geqslant 0$, as follows from Theorems 2.1 and 3.1 . 
THEOREM 3.2. Let $V \in \dot{H}^{\infty}$. Then there exists a constant $C$ independent of $k$ and $V$ such that

$$
\left\|E_{k}(k) V-E(k) V\right\|_{0} \leqslant\left\|(I-k G / 2)\left[E_{k}(k) V-E(k) V\right]\right\|_{0} \leqslant C k^{3}\|V\|_{6} .
$$

Proof. Put $V=\left[\Sigma_{m=1}^{\infty} a_{m} \phi_{m}, \Sigma_{m=1}^{\infty} b_{m} \phi_{m}, \Sigma_{m=1}^{\infty} d_{m} \phi_{m}\right]^{T}$ and for each $m=$ $1,2, \cdots$, let $y_{m}$ be the point in three-dimensional space given by

$$
y_{m}=\left[a_{m}, b_{m}, d_{m}\right]^{T} \text {. }
$$

Then, using Lemma 3.1,

$$
\begin{aligned}
& \left\|E_{k}(k) V-E(k) V\right\|_{0}^{2} \\
& =\sum_{m=1}^{\infty}\left\|e^{k \hat{G}\left(\lambda_{m}\right)} y_{m}-\left[I-(k / 2) \hat{G}\left(\lambda_{m}\right)\right]^{-1}\left[I+(k / 2) \hat{G}\left(\lambda_{m}\right)\right] y_{m}\right\|^{2} \\
& \leqslant \sum_{m=1}^{\infty}\left\|(I-k \hat{G} / 2) e^{k \hat{G}} y_{m}-(I+k \hat{G} / 2) y_{m}\right\|^{2} \\
& =\left\|(I-k G / 2)\left[E_{k}(k) V-E(k) V\right]\right\|_{0}^{2} \\
& \leqslant C k^{6}\left\{\sum_{m=1}^{\infty} \lambda_{m}^{5}\left|a_{m}\right|^{2}+\lambda_{m}^{5}\left|b_{m}\right|^{2}+\lambda_{m}^{7}\left|d_{m}\right|^{2}\right\} \\
& \leqslant C k^{6}\|V\|_{6}^{2} \text {. }
\end{aligned}
$$

This proves the theorem.

4. Some A Priori Estimates. Equations (3.1)-(3.3) define a convergent "method of lines" where only the time variable is discretized. At each time step, one must solve a coupled system of boundary value problems. We shall construct a "least-squares" procedure for approximating the problem at each time step, and eventually obtain a "fully-discrete" algorithm for the initial-boundary value problem (2.10)-(2.12). The construction rests on certain a priori estimates which we develop in the present section.

We will be dealing with arbitrary elements of the Sobolev spaces $H^{s}(\Omega)$, s a nonnegative integer. Such functions will not usually belong to $\dot{H}^{s}$. We use the notation $(u, v)_{H^{s}},\|u\|_{H^{s}}$, to indicate scalar products and norms in $H^{s}(\Omega)$. We also introduce the Hilbert space $H^{s}$, of three component vectors $U=[w, v, e]^{T}$, with the norm

$$
\|U\|_{H^{s}}=\left\{c^{2}\|w\|_{H^{s+1}}^{2}+\|v\|_{H^{s}}^{2}+\frac{c^{2}}{\gamma-1}\|e\|_{H^{s+1}}^{2}\right\}^{1 / 2} .
$$

Note that by the previously mentioned equivalence of norms, if $U \in \dot{H}^{s}$, s a nonnegative integer, then

$$
c_{s}\|U\|_{\mathcal{H}^{s}} \leqslant\|U\|_{s} \leqslant C_{s}\|U\|_{\mathcal{H}^{s}},
$$

for some positive constants $c_{s}$ and $C_{s}$, independent of $U$.

Let $L_{k}^{ \pm}$denote the operators $I \pm k G / 2$ of Section 3. We have

LEMmA 4.1. Let $U$ be an arbitrary element of $H^{2}$. Let $\alpha=2 \operatorname{Max}\left\{c^{2}, 1,(\gamma-1)\right\}$. Then 


$$
\begin{aligned}
(1-\alpha k)\|U\|_{\mathcal{H}^{0}}^{2} \leqslant & \left\|L_{k}^{-} U\right\|_{\mathcal{H}^{0}}^{2}+k \int_{\partial \Omega} c^{2} v \frac{\partial(w-e)}{\partial \nu} d s \\
& +k \int_{\partial \Omega}\left(\frac{c^{2} \sigma}{\gamma-1}\right)(e+\Delta e) \frac{\partial e}{\partial \nu} d s .
\end{aligned}
$$

Proof.

$$
\begin{aligned}
\left\|L_{k}^{-} U\right\|_{H^{0}}^{2}= & c^{2}\|w-(k / 2) v\|_{H^{1}}^{2}+\left\|c^{2}(k / 2) \Delta(e-w)+v\right\|_{L^{2}}^{2} \\
& +\frac{c^{2}}{\gamma-1}\|(k / 2)(\gamma-1) v+e-(\sigma k / 2) \Delta e\|_{H^{1}}^{2} \\
(4.4) \quad & c^{2}\|w\|_{H^{1}}^{2}+\|v\|_{L^{2}}^{2}+\frac{c^{2}}{\gamma-1}\|e\|_{H^{1}}^{2}+\frac{c^{2} k^{2}}{4}\|v\|_{H^{1}}^{2}+\frac{c^{4} k^{2}}{4}\|\Delta e-\Delta w\|_{L^{2}}^{2} \\
& +\frac{c^{2}}{\gamma-1}\|(k / 2)(\gamma-1) v-(\sigma k / 2) \Delta e\|_{H^{1}}^{2} \\
& +c^{2} k(v,(w-e))_{H^{1}}-c^{2} k(v, \Delta(w-e))_{L^{2}}-\left(c^{2} \sigma k /(\gamma-1)\right)(e, \Delta e)_{H^{1}} .
\end{aligned}
$$

Hence

(4.5) $\|U\|_{\mathcal{H}^{0}}^{2}-\left\|L_{k}^{-} U\right\|_{\mathcal{H}^{0}}^{2} \leqslant c^{2} k(v, \Delta(w-e))_{L^{2}}-c^{2} k(v,(w-e))_{H^{1}}+\frac{c^{2} \sigma k}{\gamma-1}(e, \Delta e)_{H^{1}}$.

Next, we make use of Green's formula,

$$
(v, \Delta w)_{L^{2}}=\int_{\partial \Omega} v \frac{\partial w}{\partial v} d s-D(v, w)
$$

where $\nu$ is the exterior unit normal on $\partial \Omega$, and $D(v, w)$ is the Dirichlet integral

$$
D(v, w)=\int_{\Omega} \sum_{i=1}^{N} \frac{\partial v}{\partial x_{i}} \frac{\partial w}{\partial x_{i}} d x
$$

Since,

$$
\begin{aligned}
(v,(w-e))_{H^{1}} & =(v,(w-e))_{L^{2}}+D(v,(w-e)) \\
& =(v,(w-e))_{L^{2}}+\int_{\partial \Omega} v \frac{\partial}{\partial \nu}(w-e) d s-(v, \Delta(w-e))_{L^{2}}
\end{aligned}
$$

and,

$$
\begin{aligned}
(e, \Delta e)_{H^{1}} & =(e, \Delta e)_{L^{2}}+D(e, \Delta e) \\
& =\int_{\partial \Omega}(e+\Delta e) \frac{\partial e}{\partial v} d s-D(e, e)-\|\Delta e\|_{L^{2}}^{2},
\end{aligned}
$$

we obtain on using (4.8), (4.9) in (4.5),

$$
\begin{aligned}
\|U\|_{\mathcal{H}}^{2}-\left\|L_{k}^{-} U\right\|_{\mathcal{H} 0}^{2} \leqslant & c^{2} k(v,(w-e))_{L^{2}}+c^{2} k \int_{\partial \Omega} v \frac{\partial(w-e)}{\partial \nu} d s \\
& +c^{2} k \int_{\partial \Omega}\left(\frac{\sigma}{\gamma-1}\right)(e+\Delta e) \frac{\partial e}{\partial \nu} d s .
\end{aligned}
$$

Finally, 


$$
\begin{aligned}
(v,(w-e))_{L^{2}} & \leqslant 2\|v\|_{L^{2}}^{2}+\|w\|_{L^{2}}^{2}+\|e\|_{L^{2}}^{2} \\
& \leqslant 2\|v\|_{L^{2}}^{2}+\|w\|_{H^{1}}^{2}+\|e\|_{H^{1}}^{2} \leqslant \frac{\alpha}{c^{2}}\|U\|_{\mathcal{H}}^{2} 0,
\end{aligned}
$$

where $\alpha>0$ is as in (4.3).

Hence, (4.3) follows on using (4.11) in (4.10).

Lemma 4.2. Let $U$ be in $H^{2}$. Then with $\alpha$ as in (4.3),

$$
\begin{aligned}
(1-\alpha k)\left\|L_{k}^{+} U\right\|_{\mathcal{H} 0}^{2} \leqslant & (1+\alpha k)\left\|L_{k}^{-} U\right\|_{\mathcal{H} 0}^{2}+2 k \int_{\partial \Omega} c^{2} v \frac{\partial(w-e)}{\partial \nu} d s \\
& +2 k \int_{\partial \Omega}\left(\frac{\sigma c^{2}}{\gamma-1}\right)(e+\Delta e) \frac{\partial e}{\partial \nu} d s .
\end{aligned}
$$

Proof. We have

$$
\begin{aligned}
\left\|L_{k}^{+} U\right\|_{\mathcal{H}^{0}}^{2}-\left\|L_{k}^{-} U\right\|_{H^{0}}^{2}= & 2 c^{2} k(v, \Delta(w-e))_{L^{2}} \\
& +\frac{2 c^{2} \sigma k}{\gamma-1}(e, \Delta e)_{H^{1}}-2 c^{2} k(v,(w-e))_{H^{1}} .
\end{aligned}
$$

Using (4.8), (4.9) in (4.13), we get,

$$
\begin{aligned}
\left\|L_{k}^{+} U\right\|_{H^{0}}^{2}-\| L_{k}^{-} & U \|_{H_{0}}^{2}=2 c^{2} k(v,(w-e))_{L^{2}} \\
& +2 k \int_{\partial \Omega} c^{2} v \frac{\partial(w-e)}{\partial \nu} d s+2 k \int_{\partial \Omega}\left(\frac{\sigma c^{2}}{\gamma-1}\right)(e+\Delta e) \frac{\partial e}{\partial \nu} d s .
\end{aligned}
$$

Next, as in (4.11),

$$
2 c^{2} k(v,(w-e))_{L^{2}} \leqslant 2 \alpha k\|U\|_{\mathcal{H}^{0}}^{2} .
$$

If we now use (4.15) together with Lemma 4.1 in (4.14), Lemma 4.2 follows.

5. The Fully-Discrete Problem. For the approximate solution of (3.1)-(3.3) for each $n$, we shall employ a finite-dimensional subspace, $S_{h}^{q}$, of $H^{2}$, consisting of three component vectors $\Psi$ whose entries are piecewise polynomials. The elements of $S_{h}^{q}$ will not, in general, satisfy the homogeneous boundary conditions (3.2). However, $S_{h}^{q}$ will have the property that given any three-component vector $V \in \dot{H}^{2+s} \subset H^{2}$, there is a vector $\Psi$ in $S_{h}^{q}$ such that

$$
\|V-\Psi\|_{\mathcal{H}^{l}} \leqslant C h^{s+2-l}\|V\|_{s+2}, \quad 0 \leqslant l \leqslant 2,
$$

for all $0 \leqslant s \leqslant q$. Here, $C$ is a constant independent of $V$ and $h$. The construction of such spaces is discussed in [2], [3], [4], [16], and their references. Computational investigations of least-squares procedures for elliptic boundary value problems are reported in [15]. From (5.1) we deduce that

$$
\operatorname{Inf}_{\Psi \in S_{h}^{q}} \sum_{l=0}^{2} h^{l}\|V-\Psi\|_{\mathcal{H}} \leqslant C h^{s+2}\|V\|_{s+2} .
$$

At each time step in (3.1), one must approximately solve

$$
L_{k}^{-} W=L_{k}^{+} V \text { in } \Omega
$$




$$
w_{1}=w_{3}=0 \text { on } \partial \Omega,
$$

where $V$ is known and is obtained from the preceding time step. We now introduce the bilinear form

$$
\begin{aligned}
(\Phi, \Psi)_{\Lambda}= & \left(L_{k}^{-} \Phi, L_{k}^{-} \Psi\right)_{\mathcal{H} 0}+4 k^{6} \int_{\partial \Omega}\left[\frac{\partial \phi_{1}}{\partial \nu} \frac{\partial \psi_{1}}{\partial \nu}+\frac{\partial \phi_{3}}{\partial \nu} \frac{\partial \psi_{3}}{\partial \nu}\right] d s \\
& +\pi_{k h} \int_{\partial \Omega}\left(\frac{c^{2} \sigma}{\gamma-1}\right)^{2} \phi_{3} \psi_{3} d s+\lambda_{k h} \int_{\partial \Omega} c^{4} \phi_{2} \psi_{2} \\
& +\beta_{k h} \int_{\partial \Omega}\left(\frac{c^{2} \sigma}{\gamma-1}\right)^{2} \Delta \phi_{3} \Delta \psi_{3} d s,
\end{aligned}
$$

where $\pi_{k h}, \beta_{k h}, \lambda_{k h}$ are weights which will be chosen later. See (5.19)-(5.21) below. We always assume that

$$
\pi_{k h}, \beta_{k h}, \lambda_{k h} \geqslant 1 / k^{4} \text {. }
$$

The bilinear form (5.5) is well defined for all $\Phi, \Psi$ in the Hilbert space $H^{2}$. Indeed, if $\Phi$ is in $H^{2}$, its third component, $\phi_{3}$, lies in the Sobolev space $H^{3}(\Omega)$, and the trace theorems (see e.g. [4, Section 4]) guarantee that $\partial \phi_{3} / \partial \nu, \Delta \phi_{3}$ exist and belong to $L^{2}(\partial \Omega)$.

On $H^{2}$, the form (5.5) defines an additional scalar product. That is, $\|\Phi\|_{\Lambda}=$ $\left\{(\Phi, \Phi)_{\Lambda}\right\}^{1 / 2}$ is a proper norm on $H^{2}$ for all sufficiently small $k$. This follows from Lemma 4.1 and (5.6), on using inequalities such as

$$
k \int_{\partial \Omega} c^{2} \phi_{2} \frac{\partial \phi_{3}}{\partial \nu} d s \leqslant \frac{k^{6}}{2} \int_{\partial \Omega}\left|\frac{\partial \phi_{3}}{\partial \nu}\right|^{2} d s+\frac{1}{2 k^{4}} \int_{\partial \Omega} c^{4}\left|\phi_{2}\right|^{2} d s,
$$

etc.

Lemma 5.1. Let $V \in \dot{H}^{2}$ and let $W$ be the corresponding solution of (5.3), (5.4). Then, there is a unique element $\widetilde{U}$ in $S_{h}^{q}$ minimizing $\|\Phi-W\|_{\Lambda}$ over all $\Phi$ in $S_{h}^{q}$. It is given by the equations

$$
(\widetilde{U}, F)_{\Lambda}=\left(L_{k}^{+} V, L_{k}^{-} F\right)_{\mathcal{H}^{0}}+4 k^{6} \int_{\partial \Omega}\left[\frac{\partial w_{1}}{\partial \nu} \frac{\partial f_{1}}{\partial \nu}+\frac{\partial w_{3}}{\partial \nu} \frac{\partial f_{3}}{\partial \nu}\right] d s,
$$

$\forall F$ in $S_{h}^{q}$, where $w_{i}, f_{i}$ are the ith components of $W$ and $F$, respectively.

Proof. By the Pythagorean theorem, the unique $\widetilde{U}$ in $S_{h}^{q}$ minimizing $\|\Phi-W\|_{\Lambda}$ satisfies

$$
(\widetilde{U}, F)_{\Lambda}=(W, F)_{\Lambda} \quad \forall F \text { in } S_{h}^{q}
$$

Now if $V$ is in $\dot{H}^{2}$, then, by Theorem 3.1, so is $W$. Thus $W \in \dot{H}^{3} \times \dot{H}^{2} \times \dot{H}^{3}$. Hence, $w_{1}=w_{2}=w_{3}=0$ on $\partial \Omega$ and $\Delta w_{1}=\Delta w_{3}=0$ on $\partial \Omega$. Consequently, from (5.5),

$$
(W, F)_{\Lambda}=\left(L_{k}^{-} W, L_{k}^{-} F\right)_{\mathcal{H}^{0}}+4 k^{6} \int_{\partial \Omega}\left[\frac{\partial w_{1}}{\partial \nu} \frac{\partial f_{1}}{\partial \nu}+\frac{\partial w_{3}}{\partial \nu} \frac{\partial f_{3}}{\partial \nu}\right] d s .
$$

Since $L_{k}^{-} W=L_{k}^{+} V$, the result follows.

We shall now describe a family of fully-discrete schemes for approximating the initial-boundary value problem (2.10)-(2.12). The schemes differ from one another 
only in the choice of the weights $\pi_{k h}, \lambda_{k h}$, and $\beta_{k h}$ in (5.5). We first remark that Eqs. (5.8) are not suitable for defining an approximate solution of (5.3)-(5.4), because the right-hand side of (5.8) involves unknown boundary data, namely, the normal derivatives of the solution $W$. Our strategy will be to replace these unknown derivatives by zero and to choose, as an approximation to $W$, an element of $S_{h}^{q}$ different from the $\widetilde{U}$ defined by (5.8). It is evidently crucial to be able to control the growth of the error introduced by using such wrong boundary data. This is the reason for the factor $k^{6}$ in (5.5). We have

LEMMA 5.2. Let $V \in \dot{H}^{2}$ and let $W$ be the corresponding solution of (5.3), (5.4). Let $\widetilde{U}$ be defined by (5.8), and define the approximation $\widetilde{W}$ of $W$ by the equations

$$
(\widetilde{W}, F)_{\Lambda}=\left(L_{k}^{+} V, L_{k}^{-} F\right)_{H^{0}} \quad \forall F \text { in } S_{h}^{q}, \quad \widetilde{W} \in S_{h}^{q} .
$$

Then,

$$
\|\widetilde{U}-\widetilde{W}\|_{\Lambda} \leqslant C k^{3}\|V\|_{1}
$$

Proof. We have

$$
(\widetilde{U}-\widetilde{W}, F)_{\Lambda}=4 k^{6} \int_{\partial \Omega}\left[\frac{\partial w_{1}}{\partial \nu} \frac{\partial f_{1}}{\partial \nu}+\frac{\partial w_{3}}{\partial \nu} \frac{\partial f_{3}}{\partial \nu}\right] d s \quad \forall F \text { in } S_{h}^{q} .
$$

Hence, since $\widetilde{U}-\widetilde{W} \in S_{h}^{q}$,

$$
\begin{aligned}
\|\widetilde{U}-\widetilde{W}\|_{\Lambda}^{2}= & 4 k^{6} \int_{\partial \Omega}\left[\frac{\partial w_{1}}{\partial \nu} \frac{\partial}{\partial \nu}\left(\tilde{u}_{1}-\widetilde{w}_{1}\right)+\frac{\partial w_{3}}{\partial \nu} \frac{\partial}{\partial \nu}\left(\tilde{u}_{3}-\widetilde{w}_{3}\right)\right] \\
\leqslant & 2 k^{3}\left\{\int_{\partial \Omega}\left(\left|\frac{\partial w_{1}}{\partial \nu}\right|^{2}+\left|\frac{\partial w_{3}}{\partial \nu}\right|^{2}\right) d s\right\}^{1 / 2} \\
& \cdot 2 k^{3}\left\{\int_{\partial \Omega}\left(\left|\frac{\partial}{\partial \nu}\left(\tilde{u}_{1}-\widetilde{w}_{1}\right)\right|^{2}+\left|\frac{\partial}{\partial \nu}\left(\tilde{u}_{3}-\widetilde{w}_{3}\right)\right|^{2}\right) d s\right\}^{1 / 2}
\end{aligned}
$$

on using Schwarz's inequality. Next, from (5.5),

$$
2 k^{3}\left\{\int_{\partial \Omega}\left(\left|\frac{\partial}{\partial \nu}\left(\tilde{u}_{1}-\widetilde{w}_{1}\right)\right|^{2}+\left|\frac{\partial}{\partial \nu}\left(\tilde{u}_{3}-\widetilde{w}_{3}\right)\right|^{2}\right) d s\right\}^{1 / 2} \leqslant\|\widetilde{U}-\widetilde{W}\|_{\Lambda}
$$

Therefore,

$$
\|\widetilde{U}-\widetilde{W}\|_{\Lambda} \leqslant 2 k^{3}\left\{\int_{\partial \Omega}\left(\left|\frac{\partial w_{1}}{\partial \nu}\right|^{2}+\left|\frac{\partial w_{1}}{\partial \nu}\right|^{2}\right) d s\right\}^{1 / 2} .
$$

Next, we use the trace inequality,

$$
\int_{\partial \Omega}\left|\frac{\partial w}{\partial \nu}\right|^{2} d s \leqslant C\|w\|_{H^{2}}^{2}
$$

in (5.16). This leads to

$$
\|\widetilde{U}-\widetilde{W}\|_{\Lambda} \leqslant C k^{3}\|W\|_{\mathcal{H}^{1}} \leqslant C k^{3}\|W\|_{1} .
$$

Finally, by Theorem $3.1,\|W\|_{1} \leqslant\|V\|_{1}$. This proves the lemma.

Let $\mu$ be a real number greater than 2 and let the weights $\pi_{k h}, \lambda_{k h}, \beta_{k h}$ in (5.5) satisfy 


$$
\begin{aligned}
& 1 / k^{4} \leqslant \pi_{k h} \leqslant C k^{2} h^{-2 \mu}, \\
& 1 / k^{4} \leqslant \lambda_{k h} \leqslant C k^{2} h^{-2 \mu+2}, \\
& 1 / k^{4} \leqslant \beta_{k h} \leqslant C k^{2} h^{-2 \mu+4} .
\end{aligned}
$$

For each choice of $\mu$, the fully-discrete algorithm for the initial-boundary value problem (2.10)-(2.12) is as follows. Given any initial data $W^{0}$ in $\dot{H}^{2}$, we define the sequence $\left\{\widetilde{W}^{n}\right\}$, of approximate solutions at time $t=n k$, of (3.1)-(3.3) by means of the marching procedure

$$
\begin{gathered}
\left(\widetilde{W}^{n+1}, F\right)_{\Lambda}=\left(L_{k}^{+} \widetilde{W}^{n}, L_{k}^{-} F\right)_{H^{0}} \quad \forall F \text { in } S_{h}^{q}, \quad n=0,1,2,3, \cdots . \\
\widetilde{W}^{0}=W^{0},
\end{gathered}
$$

with $\widetilde{W}^{n+1}$ being sought in $S_{h}^{q}$. Thus, given a basis for $S_{h}^{q}$, finding $\widetilde{W}^{n}$ necessitates solving a system of linear equations for the coefficients. This system always has a unique solution because $S_{h}^{q}$ is finite-dimensional and \|\|$_{\Lambda}$ is a norm on $S_{h}^{q}$. It should be noted that the scheme is defined only for initial data in $\dot{H}^{2}$ rather than in the larger space $\dot{H}^{0}$, wherein the analytic problem $(2.10)-(2.12)$ is well posed. We shall comment on this phenomenon at a later stage.

With $\widetilde{W}^{n}$ as in (5.22), put $\widetilde{W}^{1}=E_{k h} W^{0}, \widetilde{W}^{n}=E_{k h} \widetilde{W}^{n-1}=E_{k h}^{n} W^{0}$. We shall now show that the fully-discrete schemes (5.22) are unconditionally stable, that is, the family of discrete solution operators $\left\{E_{k h}^{n}\right\}$ is bounded in an appropriate norm, uniformly, as $k, h \rightarrow 0, n \rightarrow \infty, n k \leqslant T$. This is

Lемма 5.3. For all $W^{0}$ in $\dot{H}^{2}$ and sufficiently small $k$ with $0 \leqslant n k \leqslant T$, we have, independently of $h$ as $h \rightarrow 0$,

$$
\left\|E_{k h}^{n} W^{0}\right\|_{\mathcal{H}^{0}} \leqslant C e^{\alpha T}\left\|W^{0}\right\|_{\Lambda} .
$$

Proof. By (5.22) and Schwarz's inequality,

$$
\left\|\widetilde{W}^{n+1}\right\|_{\Lambda}^{2} \leqslant\left\|L_{k}^{+} \widetilde{W}^{n}\right\|_{\mathcal{H}^{0}}\left\|L_{k}^{-} \widetilde{W}^{n+1}\right\|_{\mathcal{H}^{0}} \leqslant\left\|L_{k}^{+} \widetilde{W}^{n}\right\|_{\mathcal{H}^{0}}\left\|\widetilde{W}^{n+1}\right\|_{\Lambda} .
$$

Hence, $\left\|\widetilde{W}^{n+1}\right\|_{\Lambda}^{2} \leqslant\left\|L_{k}^{+} \widetilde{W}^{n}\right\|_{\mathcal{H}}^{2}$. Next, from (4.12) and (5.5), (5.6),

$$
\left\|L_{k}^{+} \widetilde{W}^{n}\right\|_{\mathcal{H}^{0}}^{2} \leqslant\left(\frac{1+\alpha k}{1-\alpha k}\right)\left\|\widetilde{W}^{n}\right\|_{\Lambda}^{2}
$$

so that

$$
\left\|\widetilde{W}^{n+1}\right\|_{\Lambda}^{2} \leqslant\left(\frac{1+\alpha k}{1-\alpha k}\right)\left\|W^{n}\right\|_{\Lambda}^{2}
$$

Hence, if $0 \leqslant(n+1) k \leqslant T$,

$$
\left\|E_{k h}^{n+1} W^{0}\right\|_{\Lambda} \leqslant C e^{\alpha T}\left\|W^{0}\right\|_{\Lambda}
$$

Finally, from (4.3) and (5.5), (5.6),

$$
\left\|E_{k h}^{n+1} W^{0}\right\|_{\mathcal{H}^{0}} \leqslant \frac{1}{1-\alpha k}\left\|E_{k h}^{n+1} W^{0}\right\|_{\Lambda} .
$$

This proves the lemma. 
LemmA 5.4. Let $0 \leqslant s \leqslant q$ and let $W \in \dot{H}^{2+s}$. Fix $\mu>2$ and let (5.19)-(5.21) be satisfied. Then there is a constant $C$ independent of $W$ and $h$ such that,

$$
\operatorname{Inf}_{\Phi \in S_{h}^{q}}\|\Phi-W\|_{\Lambda} \leqslant C k h^{s+2-\mu}\|W\|_{2+s}
$$

Proof. We have for any $U=[w, v, e]^{T}$ in $H^{2}$,

$$
\begin{aligned}
\|U\|_{\Lambda}^{2}= & \left\|L_{k}^{-} U\right\|_{\mathcal{H}^{0}}^{2}+4 k^{6} \int_{\partial \Omega}\left(\left|\frac{\partial w}{\partial \nu}\right|^{2}+\left|\frac{\partial e}{\partial \nu}\right|^{2}\right) d s \\
& +\left(\frac{c^{2} \sigma}{\gamma-1}\right)^{2} \pi_{k h} \int_{\partial \Omega}|e|^{2} d s+\left(\frac{c^{2} \sigma}{\gamma-1}\right)^{2} \beta_{k h} \int_{\partial \Omega}|\Delta e|^{2} d s \\
& +c^{4} \lambda_{k h} \int_{\partial \Omega}|v|^{2} d s .
\end{aligned}
$$

From (4.4),

$$
\begin{aligned}
\left\|L_{k}^{-} U\right\|_{H^{0}}^{2} & \leqslant C\|U\|_{\mathcal{H}^{0}}^{2}+C k^{2}\left\{\|v\|_{H^{1}}^{2}+\|\Delta e\|_{L^{2}}^{2}+\|\Delta w\|_{L^{2}}^{2}\right\}+C k^{2}\|\Delta e\|_{H^{1}}^{2} \\
& \leqslant C\|U\|_{\mathcal{H}^{0}}^{2}+C k^{2}\|U\|_{\mathcal{H}^{1}}^{2}+C k^{2}\|U\|_{\mathcal{H}^{2}}^{2} .
\end{aligned}
$$

To estimate the boundary integrals in (5.31) we use the inequalities,

$$
\begin{gathered}
\int_{\partial \Omega}\left(\left|\frac{\partial w}{\partial \nu}\right|^{2}+\left|\frac{\partial e}{\partial \nu}\right|^{2}\right) d s \leqslant C\|w\|_{H^{2}}^{2}+C\|e\|_{H^{2}}^{2} \leqslant C\|U\|_{\mathcal{H}^{1}}^{2}, \\
\int_{\partial \Omega}|e|^{2} d s \leqslant C\|e\|_{\mathcal{H}^{1}}^{2} \leqslant C\|U\|_{\mathcal{H}^{0}}^{2}, \\
\int_{\partial \Omega}|\Delta e|^{2} d s \leqslant C\|e\|_{\mathcal{H}^{3}}^{2} \leqslant C\|U\|_{\mathcal{H}^{2}}^{2}, \\
\int_{\partial \Omega}|v|^{2} d s \leqslant C\|v\|_{\mathcal{H}^{1}}^{2} \leqslant C\|U\|_{\mathcal{H}^{1}}^{2} .
\end{gathered}
$$

Hence,

$$
\begin{aligned}
\|U\|_{\Lambda}^{2} & \leqslant C\left\{\left(1+\pi_{k h}\right)\|U\|_{\mathcal{H} 0}^{2}+\left(k^{2}+k^{6}+\lambda_{k h}\right)\|U\|_{\mathcal{H} 1}^{2}+\left(k^{2}+\beta_{k h}\right)\|U\|_{\mathcal{H}^{2}}^{2}\right\} \\
& \leqslant C\left\{\pi_{k h}\|U\|_{\mathcal{H}}^{2}+\lambda_{k h}\|U\|_{\mathcal{H}^{1}}^{2}+\beta_{k h}\|U\|_{\mathcal{H}^{2}}^{2}\right\} \\
& \leqslant C k^{2} h^{-2 \mu}\left\{\|U\|_{\mathcal{H} 0}^{2}+h^{2}\|U\|_{\mathcal{H} 1}^{2}+h^{4}\|U\|_{\mathcal{H}^{2}}^{2}\right\}
\end{aligned}
$$

and

$$
\|U\|_{\Lambda} \leqslant C k h^{-\mu}\left\{\sum_{l=0}^{2} h^{l}\|U\|_{\mathcal{H} l}\right\} .
$$

From (5.38) and (5.2), we have,

as required.

$$
\operatorname{Inf}_{\Phi \in S_{h}^{q}}\|\Phi-W\|_{\Lambda} \leqslant C k h^{s+2-\mu}\|W\|_{2+s}
$$

Recall that $E(t)$ is the solution operator for the analytic problem (2.10)-(2.12), $E_{k}(t)$ that for the semidiscrete problem (3.1)-(3.3), and $E_{k h}(t)=E_{k h}^{n}$ is the fully-discrete solution operator. In Lemma 5.3, the stability of the fully-discrete problem was established. The next lemma shows that the fully-discrete scheme is consistent with (2.10)(2.12). 
Lemma 5.5. Fix $\mu>2$ in (5.19)-(5.21) and let $q \geqslant \mu-2>0$ in (5.1). Let $V \in \dot{H}^{\infty}$. Then

$$
\left\|E_{k h} V-E(k) V\right\|_{\Lambda} \leqslant C\left\{k h^{q+2-\mu}\|V\|_{2+q}+k^{3}\|V\|_{1}+k^{3}\|V\|_{6}\right\} .
$$

Proof. Since $V \in \dot{H}^{2}, \widetilde{U}$ defined by (5.8) minimizes $\left\|\Phi-E_{k}(k) V\right\|_{\Lambda}$ over $S_{h}^{q}$. Hence, using Lemma 5.4 and Theorem 3.1,

$$
\left\|\widetilde{U}-E_{k}(k) V\right\|_{\Lambda} \leqslant C k h^{q+2-\mu}\left\|E_{k}(k) V\right\|_{2+q} \leqslant C k h^{q+2-\mu}\|V\|_{2+q} .
$$

Next, $\widetilde{W}=E_{k h} V$, defined by (5.11), satisfies (5.12). Hence,

$$
\left\|E_{k h} V-E_{k}(k) V\right\|_{\Lambda} \leqslant C\left(k h^{q+2-\mu}\|V\|_{2+q}+k^{3}\|V\|_{1}\right) .
$$

From Theorem 3.2,

$$
\left\|L_{k}^{-}\left(E(k) V-E_{k}(k) V\right)\right\|_{H_{0}} \leqslant C k^{3}\|V\|_{6} .
$$

From (5.5), since $V \in \dot{H}^{2}$,

$$
\begin{aligned}
\left\|E(k) V-E_{k}(k) V\right\|_{\Lambda}^{2} \leqslant & \leqslant L_{k}^{-}\left(E(k) V-E_{k}(k) V \|_{\mathcal{H}^{0}}^{2}\right. \\
& +4 k^{6} \int_{\partial \Omega}\left|\frac{\partial}{\partial \nu}\left(E(k) V-E_{k}(k) V\right)_{1}\right|^{2}+\left|\frac{\partial}{\partial \nu}\left(E(k) V-E_{k}(k) V\right)_{3}\right|^{2} d s \\
& \leqslant \| L_{k}^{-}\left(E(k) V-E_{k}(k) V\left\|_{\mathcal{H}^{0}}^{2}+C k^{6}\right\| E(k) V\left\|_{1}^{2}+C k^{6}\right\| E_{k}(k) V \|_{1}^{2} .\right.
\end{aligned}
$$

Hence, using (5.43) and Theorems 2.1 and 3.1,

$$
\left\|E(k) V-E_{k}(k) V\right\|_{\Lambda} \leqslant C k^{3}\left(\|V\|_{1}+\|V\|_{6}\right) .
$$

The lemma now follows from (5.45), (5.42) and the triangle inequality.

We may now state and prove the following theorem concerning the convergence of each of the schemes (5.22), (5.23).

Theorem 5.1. Fix $\mu>2$ in (5.19)-(5.21) and let $q \geqslant \mu-2>0$ in (5.1). Let $W^{0} \in \dot{H}^{\mathrm{Max}(2+q, 6)}$. Then, with $\alpha$ as in (4.3), we have for all $0 \leqslant t=n k \leqslant T$,

$$
\begin{aligned}
\left\|E_{k h}(t) W^{0}-E(t) W^{0}\right\|_{\mathcal{H}^{0}} & \leqslant C\left\|E_{k h}(t) W^{0}-E(t) W^{0}\right\|_{\Lambda} \\
& \leqslant C T e^{\alpha T}\left\{h^{q+2-\mu}\left\|W^{0}\right\|_{2+q}+k^{2}\left\|W^{0}\right\|_{6}\right\} .
\end{aligned}
$$

Proof. We use the identity

$$
E_{k h}(t) W^{0}-E(t) W^{0}=\sum_{j=0}^{n} E_{k h}^{n-1-j}\left[E_{k h}-E(k)\right] E(j k) W^{0} .
$$

By Lemma 5.5 and Theorem 2.1,

$$
\begin{aligned}
& \left\|E_{k h} E(j k) W^{0}-E(k) E(j k) W^{0}\right\|_{\Lambda} \\
& \quad \leqslant C k\left\{h^{q+2-\mu}\left\|E(j k) W^{0}\right\|_{2+q}+k^{2}\left\|E(j k) W^{0}\right\|_{1}+k^{2}\left\|E(j k) W^{0}\right\|_{6}\right\} \\
& \leqslant C k\left\{h^{q+2-\mu}\left\|W^{0}\right\|_{2+q}+k^{2}\left\|W^{0}\right\|_{6}\right\} .
\end{aligned}
$$

Next, by Lemma 5.3, $\left\|E_{k h}^{n}\right\|_{\Lambda} \leqslant C e^{\alpha T}$. Hence, from (5.47), (5.48), 


$$
\left\|E_{k h}(t) W^{0}-E(t) W^{0}\right\|_{\Lambda} \leqslant C T e^{\alpha T}\left\{h^{q+2-\mu}\left\|W^{0}\right\|_{2+q}+k^{2}\left\|W^{0}\right\|_{6}\right\} .
$$

Finally, from (4.3),

$$
\left\|E_{k h}(t) W^{0}-E(t) W^{0}\right\|_{\mathcal{H}^{0}} \leqslant \frac{1}{1-\alpha k}\left\|E_{k h}(t) W^{0}-E(t) W^{0}\right\|_{\Lambda} .
$$

The theorem follows from (5.49), (5.50).

Important Remarks. The assumptions (5.19)-(5.22) about the weights $\pi_{k h}, \lambda_{k h}$ and $\gamma_{k h}$, require that

$$
h \leqslant C k^{3(\mu-2)} \text { as } k, h \rightarrow 0 .
$$

This requirement is a consistency condition rather than a stability condition. As a consequence of this requirement, the error bound (5.46) has the form

$$
\left\|E_{k h}(t) W^{0}-E(t) W^{0}\right\|_{\mathcal{H}} \leqslant C T e^{\alpha T}\left\{k^{2}\left\|W^{0}\right\|_{6}+k^{(3 q-3 \mu+6) /(\mu-2)}\left\|W^{0}\right\|_{2+q}\right\} .
$$

Thus, the smallest possible error is $O\left(k^{2}\right)$ and it occurs if $\mu$ and $q$ are such that

$$
q=5(\mu-2) / 3 \text {. }
$$

Assume now that the initial data $W^{0}$ are sufficiently smooth. Given any positive integer value of $q$, we see from (5.52), (5.53) that we get $O\left(k^{2}\right)$ accuracy by choosing $\mu$ in (5.19) $-(5.22)$ so that

$$
\mu=(3 q+10) / 5 .
$$

Thus, for example, if we choose $q=1$ in (5.1) and let $S_{h}^{q}$ be the space of three-component vectors where the first and third components are cubic splines, and the second is a quadratic spline, we obtain $O\left(k^{2}\right)$ accuracy in the $H_{0}^{1} \times L^{2} \times H_{0}^{1}$ norm on choosing $\mu=13 / 5$. Notice however that this means $h$ must be chosen so that

$$
h \leqslant C k^{5} \quad \text { as } h, k \rightarrow 0 .
$$

Such a constraint on $h$ is obviously impractical from the computational standpoint. We elect instead to use larger values of $q$, in order to obtain $O\left(k^{2}\right)$ accuracy with a more favorable mesh inequality (5.51). Thus, with $\mu=q=5$ and $S_{h}^{q}$ chosen to be splines of degree $7 \times 6 \times 7$, we get $O\left(k^{2}\right)$ accuracy with $h$ satisfying

$$
h \leqslant C k \quad \text { as } k, h \rightarrow 0 .
$$

Other combinations of $q$ and $\mu$ which yield $O\left(k^{2}\right)$ accuracy under sufficient smoothness of $W^{0}$, are listed in the following table.

The nature of the penalty which must be paid in order to be able to use the wrong values for the normal derivatives of the solution in the fully discrete scheme, is now clear. In the Crank-Nicolson least-squares scheme for the heat equation discussed in [4], one has $h \leqslant C k^{2 / 3}$ so that quartic splines are needed to obtain $O\left(k^{2}\right)$ accuracy in the $L^{2}$ norm. Here, using a comparable mesh inequality, i. e., $h \leqslant C k^{5 / 7}$, we see from Table 1 that we need splines of degree $9 \times 8 \times 9$ to obtain $O\left(k^{2}\right)$ accuracy in the $H_{0}^{1} \times L^{2} \times H_{0}^{1}$ norm. 
TABLE 1

\begin{tabular}{cccl}
$\begin{array}{c}\text { Degree of Splines } \\
\text { in } S_{h}^{q}\end{array}$ & $q$ & $\mu$ & Mesh Inequality \\
\hline $4 \times 3 \times 4$ & 2 & $16 / 5$ & $h \leqslant C k^{5 / 2}$ \\
$5 \times 4 \times 5$ & 3 & $19 / 5$ & $h \leqslant C k^{5 / 3}$ \\
$6 \times 5 \times 6$ & 4 & $22 / 5$ & $h \leqslant C k^{5 / 4}$ \\
$7 \times 6 \times 7$ & 5 & 5 & $h \leqslant C k$ \\
$8 \times 7 \times 8$ & 6 & $28 / 5$ & $h \leqslant C k^{5 / 6}$ \\
$9 \times 8 \times 9$ & 7 & $3 / 5$ & $h \leqslant C k^{5 / 7}$ \\
$10 \times 9 \times 10$ & 8 & $34 / 5$ & $h \leqslant C k^{5 / 8}$ \\
$11 \times 10 \times 11$ & 9 & $37 / 5$ & $h \leqslant C k^{5 / 9}$ \\
$12 \times 11 \times 12$ & 10 & 8 & $h \leqslant C k^{1 / 2}$ \\
\hline
\end{tabular}

The above remarks concerning $O\left(k^{2}\right)$ accuracy assume that $W^{0}$ is sufficiently smooth, i.e., $W^{0} \in \dot{H}^{\operatorname{Max}(q+2,6)}$. More generally, we have

THEOREM 5.2. Let $W^{0} \in \dot{H}^{s}, s \geqslant 2$. Choose a positive integer $q$ in (5.1), and let $\mu$ in (5.19)-(5.21) satisfy (5.54). Then for $0 \leqslant t \leqslant T$,

$$
\left\|E_{k h}(t) W^{0}-E(t) W^{0}\right\|_{H^{0}} \leqslant C \exp (\alpha T)\left\{k^{2 \operatorname{Min}(1,(s-2) / q,(s-2) / 4)}\right\}\left\|W^{0}\right\|_{s} .
$$

Proof. If $W^{0}=[w, v, e]^{T} \in \dot{H}^{2}$, then

$$
\begin{aligned}
\left\|W^{0}\right\|_{\Lambda}^{2} & =\left\|L_{k}^{-} W^{0}\right\|_{H 0}^{2}+4 k^{6} \int_{\partial \Omega}\left(\left|\frac{\partial w}{\partial v}\right|^{2}+\left|\frac{\partial e}{\partial \nu}\right|^{2}\right) d s \\
& \leqslant C\left\{\left\|W^{0}\right\|_{0}^{2}+\left(k^{2}+k^{6}\right)\left\|W^{0}\right\|_{1}^{2}+k^{2}\left\|W^{0}\right\|_{2}^{2}\right\} \leqslant C\left\|W^{0}\right\|_{2}^{2} .
\end{aligned}
$$

From Lemma 5.3, Theorem 2.1, (5.58), and the triangle inequality,

$$
\left\|E_{k h}(t) W^{0}-E(t) W^{0}\right\|_{\mathcal{H}^{0}} \leqslant C e^{\alpha T}\left\|W^{0}\right\|_{2} .
$$

If $W^{0} \in \mathcal{H}^{\mathrm{Max}(q+2,6)}$, then with $\mu$ as in (5.54), we have from (5.52)

$$
\left\|E_{k h}(t) W^{0}-E(t) W^{0}\right\|_{\mathcal{H}^{0}} \leqslant C T \exp (\alpha T)\left\{k^{2}\left\|W^{0}\right\|_{\mathrm{Max}(q+2,6)}\right\} .
$$

Next, the spaces $\dot{H}^{s}$ have the interpolation property discussed in [4, Lemma 2.2]. Hence, if $W^{0} \in \dot{H}^{s}, s \geqslant 2$,

$$
\left\|E_{k h}(t) W^{0}-E(t) W^{0}\right\|_{\mathcal{H}^{0}} \leqslant C \exp (\alpha T)\left\{k^{2 \operatorname{Min}(1,(s-2) / q,(s-2) / 4)}\right\}\left\|W^{0}\right\|_{s} .
$$

Remark. While the analytic problem (2.10)-(2.12) is well posed in $\dot{H}^{0}$, the fullydiscrete scheme requires $W^{0} \in \dot{H}^{2}$ in order to obtain convergence. A similar phenomenon occurs in [4]. For initial data in $\dot{H}^{s}, 0 \leqslant s \leqslant 2$, a convergent least-squares procedure can be constructed based on the pure implicit scheme, rather than the CrankNicolson. For the case of the Dirichlet problem for the wave equation, such a procedure 
is discussed in [5], and similar difficulties are encountered in connection with unknown normal derivatives.

Department of Mathematics and Statistics

The University of New Mexico

Albuquerque, New Mexico 87131

1. W. F. AMES, Numerical Methods for Partial Differential Equations, Barnes and Noble, New York, 1969. MR 41 \#7862.

2. G. BIRKHOFF, M. H. SCHULTZ \& R. S. VARGA, "Piecewise Hermite interpolation in one and two variables with applications to partial differential equations," Numer. Math., v. 11, 1968, pp. 232-256. MR 37 \#2404.

3. J. H. BRAMBLE \& S. R. HILBERT, "Estimation of linear functionals on Sobolev spaces with application to Fourier transforms and spline interpolation," SIAM J. Numer. Anal., v. 7, 1970, pp. 112-124. MR 41 \#7819.

4. J. H. BRAMBLE \& V. THOMÉE, "Semidiscrete-least squares methods for a parabolic boundary value problem," Math. Comp., v. 26, 1972, pp. 633-648.

5. A. CARASSO, "A least squares procedure for the wave equation," Math. Comp., v. 28, 1974, pp. 757-767.

6. F. HARLOW \& A. AMSDEN, Fluid Dynamics, LASL Monograph LA 4700, Los Alamos Scientific Laboratories, Los Alamos, N. M., 1971.

7. J. L. LIONS, "Sur l'approximation de la solution d'équations d'évolution couplées, Rend. Mat., v. 1, 1968, pp. 141-176. MR 39 \#655.

8. J. L. LIONS, "On the numerical approximation of some equations arising in hydrodynamics," Numerical Solution of Field Problems in Continuum Physics, SIAM-AMS Proc., vol. 2, Amer.

Math. Soc., Providence, R.I., 1970, pp. 11-23. MR 41 \#5796.

9. J. L. LIONS \& P. A. RAVIART, "Remarques sur la résolution et l'approximation d'équations d'évolution couplées," I. C. C. Bull., v. 5, 1966, pp. 1-21. MR 34 \#4650.

10. J. L. LIONS \& E. MAGENES, Problèmes aux Limites non Homogènes et Applications, vol. 1, Dunod, Paris, 1968. MR 40 \#512.

11. H. MORIMOTO, "Stability in the wave equation coupled with heat flow," Numer. Math., v. 4, 1962, pp. 136-145. MR 27 \#4385.

12. R. D. RICHTMYER \& K. W. MORTON, Stability Studies for Difference equations. I. Non-Linear Instability. II. Coupled Sound and Heat Flow, Report NYO 1480-5, Courant Inst. of Math. Sci., New York Univ., New York, 1964.

13. R. D. RICHTMYER \& K. W. MORTON, Difference Methods for Initial-Value Problems, 2nd ed., Interscience, New York, 1967. MR 36 \#3515.

14. C. A. ROUSE, "A method for the numerical calculation of hydrodynamic flow and radiation diffusion by implicit differencing," J. Soc. Indust. Appl. Math., v. 9, 1961, pp. 127-135. MR 23 \#B2593.

15. S. M. SERBIN, Doctorial Dissertation, Cornell Univ., Ithaca, N. Y., 1972.

16. R. S. VARGA, Functional Analysis and Approximation Theory in Numerical Analysis, Regional Conference Series in Applied Mathematics, vol. 3, Soc. Indust. Appl. Math., Philadelphia, Pa., 1971.

17. B. WENDROFF, The Initial Value Problem, Lecture Notes, Department of Mathematics, University of Denver, Col., 1969. 